\title{
Tensile detwinning in bi-twinned metallic nanowires
}

\author{
Guangming Cheng ${ }^{1}$ and Yong $\mathrm{Zhu}^{2}$
}

${ }^{1}$ Princeton Institute for the Science and Technology of Materials, Princeton University, Princeton, New Jersey, United States, ${ }^{2}$ Department of Mechanical and Aerospace Engineering, North Carolina State University, Raleigh, North Carolina, United States

\section{Email: gcheng2@princeton.edu and yzhu@ncsu.edu}

Metallic NWs usually exhibit ultrahigh mechanical strength and are ideal candidates for studying fundamental deformation mechanisms at the nanoscale.[1-5] Metallic NWs with internal TBs have received much attention recently, such as metallic NWs with parallel, inclined or perpendicular TBs with respect to the NW length direction. Interaction between dislocations and TBs has become one of the most fundamental problems in materials science due to the emergence of nanotwinned materials over the past 2 decades. The twinned metallic NWs can provide an interesting platform to investigate the energetics and kinetics of dislocation-twin boundary interactions. In spite of numerous studies on mechanical behaviors of nanowires (NWs) focusing on the surface effect, there is still a general lack of understanding on how the internal microstructure of NWs influences their deformation mechanisms. Here, based on in situ transmission electron microscopy testing, we report a transition of the deformation mechanism from localized dislocation slip to delocalized plasticity via an anomalous tensile detwinning mechanism in bitwinned metallic NWs with a single twin boundary (TB) running parallel to the NW length. [6-8] The transition of the two mechanisms depends on the volume ratio between the two twin variants and the crosssectional aspect ratio, which can be explained by an energy-based criterion.[7]

Bi-twinned NWs with a single TB running parallel to the NW length direction (Fig. 1) was found to be dominant ( $81 \%$ in the examined $113 \mathrm{NWs)} \mathrm{in} \mathrm{the} \mathrm{crystalline} \mathrm{Ag} \mathrm{NWs} \mathrm{synthesized} \mathrm{by} \mathrm{physical} \mathrm{vapor}$ deposition.[6] Figure 1c shows schematically a bi-twinned NW with the axial direction of [110]. Two twin variants are distributed at the two sides of the TB with symmetrical arrangement of crystal planes such as the marked (111) and (1-11) in Fig. 1(c). It has been reported that the deformation modes in the bitwinned NWs are affected by the cross-sectional aspect ratio and the volume ratio between the two twin variants [7] ( $\mathrm{r}$ defined as Vsmall/Vlarge). Tensile detwinning deformation was identified to lead to the observed large plasticity in bi-twinned NWs with a small volume ratio of 0.19 (Fig. 1a,b), which was different from both the twinning-induced superplasticity in single-crystalline NWs [2] and the localized dislocation slip in bi-twinned NWs with large volume ratio [7]. During the tensile detwinning process, a new single crystalline phase was formed and propagate along the NW length, reorienting the NW to the [001] direction (see the cross-sectional TEM image and corresponding diffraction pattern in Fig. 1d). As shown in Fig. 1b, there was an elongation of 56.8\% measured from the two ends marked by green arrows (singlecrystalline phase) in the bi-twinned NW (Fig. 1d). The large elongation can be attributed to the crosssectional change, an area shrinkage of $36 \%$ (comparing locations A and B in Fig. 1b) during the plastic deformation, as shown in Fig. 1d. Note that there is no obvious change of the dimension along [1-10] orientation but a large size shrinkage along the direction perpendicular to [1-10] orientation (38.5\%). [9] 
a

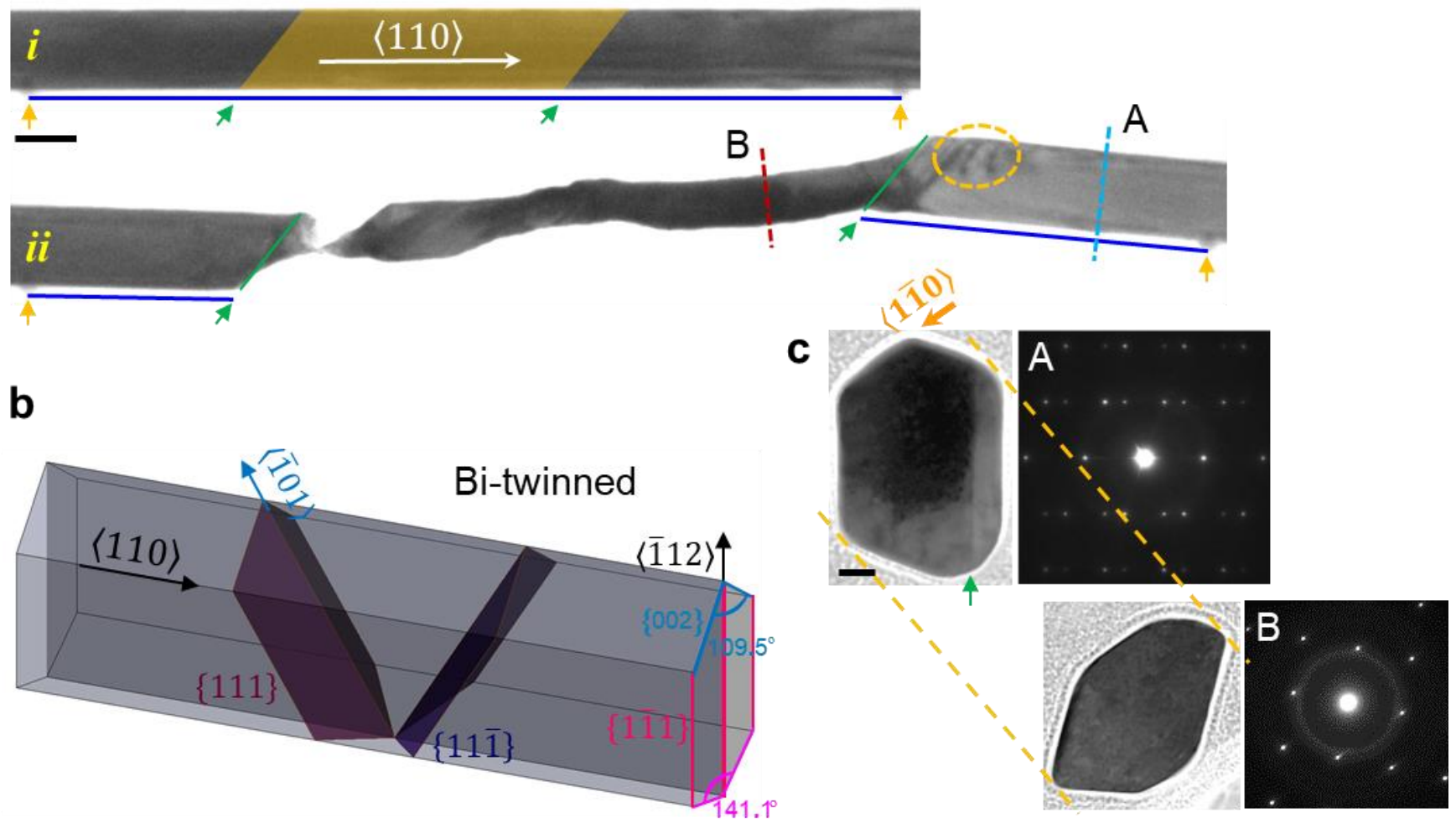

Figure 1. (a,b) Large plasticity observed in a bi-twinned NW with small volume ratio undergoing tensile detwinning deformation: (a) before tensile testing and (b) after tensile detwinning deformation. Two displacement markers for strain measurement are labeled by orange arrows. The yellow marked area in (a) correspond to the deformed parts in (b) labeled by green arrows. Localized dislocations are marked by dashed oval in (b). TEM observation is along [1-10] of the dominant twin variant as marked in (d) dashed lines in (b) marked the positions for the cross-sectional TEM images in (d). (c) Schematic drawings of bi-twinned structure with length along [110] direction. (d), Corresponding cross-sectional TEM images and diffraction patterns of the tested NW before and after plastic deformation in (a) and (b). The TB was marked by green arrow.

\section{References}

[1] S. Yin, G.M. Cheng, T.H. Chang, G. Richter, Y. Zhu, H.J. Gao, Hydrogen embrittlement in metallic nanowires, Nature Communications 10 (2019).

[2] S. Yin, G. Cheng, G. Richter, H. Gao, Y. Zhu, Transition of Deformation Mechanisms in SingleCrystalline Metallic Nanowires, Acs Nano 13(8) (2019) 9082-9090.

[3] G. Cheng, S. Yin, C. Li, T.-H. Chang, G. Richter, H. Gao, Y. Zhu, In-situ TEM study of dislocation interaction with twin boundary and retraction in twinned metallic nanowires, Acta Materialia 196 (2020) 304-312.

[4] T.-H. Chang, G. Cheng, C. Li, Y. Zhu, On the size-dependent elasticity of penta-twinned silver nanowires, Extreme Mechanics Letters 8 (2016) 177-183.

[5] Q. Qin, S. Yin, G. Cheng, X. Li, T.-H. Chang, G. Richter, Y. Zhu, H. Gao, Recoverable plasticity in penta-twinned metallic nanowires governed by dislocation nucleation and retraction, Nature Communications 6 (2015).

[6] S. Yin, G. Cheng, Y. Zhu, H. Gao, Competition between shear localization and tensile detwinning in twinned nanowires, PHYSICAL REVIEW MATERIALS 4 (2020) 023603. 
[7] G. Cheng, S. Yin, T.-H. Chang, G. Richter, H. Gao, Y. Zhu, Anomalous Tensile Detwinning in Twinned Nanowires, Physical Review Letters 19(25) (2017).

[8] G. Cheng, S. Yao, X. Sang, B. Hao, D. Zhang, Y.K. Yap, Y. Zhu, Evolution of Irradiation-Induced Vacancy Defects in Boron Nitride Nanotubes, Small 12(6) (2016) 818-824.

[9] The authors acknowledge funding from the National Science Foundation (NSF) under Award No. CMMI-1030637 and No. 1301193.and the use of the Analytical Instrumentation Facility (AIF) at North Carolina State University (NSF Award No. ECCS-1542015). 